\title{
An Improved Flexibility Metric Based on Kernel Density Estimators Applied on the Greek Power System
}

\author{
Konstantinos F. Krommydas ${ }^{1,2(\bowtie)}$, Akylas C. Stratigakos ${ }^{1,2}$, \\ Christos Dikaiakos ${ }^{2}$, George P. Papaioannou ${ }^{2}$, Elias Zafiropoulos ${ }^{3}$, \\ and Lambros Ekonomou ${ }^{3}$ \\ ${ }^{1}$ Department of Electrical and Computer Engineering, \\ University of Patras, 26504 Rion, Patras, Greece \\ krommydas@ece.upatras.gr \\ 2 Research, Technology and Development Department, \\ Independent Power Transmission Operator (IPTO) S.A., \\ 89 Dyrrachiou \& Kifisou Str. Gr, 10443 Athens, Greece \\ 3 Institute of Communications and Computer Systems, \\ 9 Iroon Polytechniou Street, 15780 Athens, Greece
}

\begin{abstract}
The large-scale integration of variable renewable energy (VRE) in power systems, such as wind and solar, increases the flexibility needed to maintain the load-generation balance. In order for the power operators to plan for secure and reliable operation, they must examine whether there exists sufficient power system flexibility to meet ramps caused by the increased VRE integration and the system demand. In this context, the paper aims to propose an improved flexibility metric to accurately evaluate the flexibility level of a power system in the planning stage. The proposed metric is based on kernel density estimators and expresses the probability of the flexibility residual (the difference between the available flexibility and the expected net load ramps) being less than zero. The Greek power system is used as case study in order to evaluate the proposed index. In particular, the unit commitment optimization problem with flexibility constraints for ten different scenarios based on the ENTSO-E methodology for the time period 2020-2024 is solved and then the proposed metric is calculated. Finally, this index is compared to the well known insufficient ramping resource expectation (IRRE) metric to further evaluate it.
\end{abstract}

Keywords: Flexibility metrics $\cdot$ Unit commitment problem $\cdot$ Kernel function

\section{Introduction}

The increasing penetration of variable renewable energy (VRE) presents significant technical challenges for balancing the power system supply and demand. More specifically, as the integration of VRE increases, the net load, which is the difference between electrical load and renewable power becomes more variable with steeper ramps, shorter peaks, and lower turn-downs than the load itself [1]. Therefore, system flexibility or the ability of a system to meet changes in demand and VRE production plays a crucial role [2]. 
Until now, traditional capacity adequacy studies focused on the amount capacity available, the forced outage rate of each resource and the system demand. In these studies, metrics such as loss of expectation (LOLE) [3, 4], the expected energy not served (EENS) or well-being analysis [5] are standard measures by which a planned portfolio is evaluated and have served the industry well until now. However, as the shares of VRE increases, the planning approach will need to change and should incorporate new metrics and additional factors, such as the availability and ramp rate of the resources, start-up time and minimum stable operation of the conventional generating units, the variability and uncertainty of the net load and reserve provision planning. But assessing flexibility is not yet common practice. Although indirectly involved in the planning of most power systems, such assessments do not yet follow a comprehensive, transferable methodology that might facilitate common practice among operators. It is a highly complex process considering many operation and design aspects [6].

In this frame, different flexibility studies and metrics have been presented in the literature that focus on the long-term planning of power systems. In [7] a methodology is developed to deal with a significant degree of uncertainty about time and location of generating assets expansion and in [8] a method is proposed for evaluating the system flexibility to adapt quickly with limited costs to every change from the initial planning conditions, with a particular regard to the changes in generation. In [9] a flexibility metric is constructed that reflects the largest variation range uncertainty that the system can sustain by using the four-element framework (time, uncertainty, action and cost), while in [10] the insufficient ramping resource expectation (IRRE) metric is proposed to measure power system flexibility for use in long-term planning and is derived from traditional generation adequacy metrics. In [11] the metric of the number of periods of flexibility deficit (PFD) is proposed to characterize the flexibility of a system.

Taking into account the new flexibility metrics and approaches presented in the literature, the conventional adequacy studies of the Greek power system are revisited by taking into account flexibility requirements, resources and metrics. In particular, considering ten different scenarios for the Greek net load for the time period 2020 2024 that are determined based on the ENTSO-E methodology, we solve the unit commitment optimization problem by including both system power balance constraints and ramp capability constraints of the flexible resources. Furthermore, the flexibility metric IRRE is calculated, which expresses the number of up and down ramps for which there would be insufficient ramping resources available. Calculation of the IRRE follows a similar structure to the LOLE, however, it targets on the unavailability of flexibility and not on the shortage of generation capacity.

However, the IRRE metric does not take into account the temporal correlation between the flexibility available and the flexibility required which may lead to misleading results about the system's flexibility. For example at the highest net load levels, most generator would be at high output and available to offer the additional downward flexibility likely to be required by the system, therefore IRRE underestimates the system flexibility which can lead to incorrect planning and unnecessary investments from the system operator.

In order to overcome this drawback, we utilize the PFD index approach. In particular, we calculate the flexibility residual, which is the difference between the 
available flexibility and the expected net load ramps, for each observation and horizon and then determine the probability of the flexibility residual distribution being less than zero. However, in contrast to the PFD approach, we use a non-parametric kernel distribution to estimate the probability distribution function, which can provide more accurate and robust results. Furthermore, the proposed metric incorporates the temporal correlation between the flexibility available and the flexibility required in comparison to the IRRE index. Overall, the main contribution of this work can be summarized as follows:

1. The traditional adequacy studies of the Greek power system are upgraded by adding the flexibility requirements and flexibility resources.

2. Using a Kernel density estimator we estimate the flexibility of the system over different time horizons and directions and highlight the time horizons of net load ramping in which the system is most vulnerable.

3. Simulations that use real data for both the flexibility requirements and flexibility resources are performed for solving the unit commitment optimization problem with flexibility requirements constraints of the Greek power system for the time period 2020-2024.

The rest of the paper is organized as follows. In Sect. 2, some preliminaries in flexibility of power systems are introduced. In Sect. 3, the flexibility metrics that are used in the flexibility assessment study of Greece are presented. In Sect. 4, the unit commitment problem is formulated as a mixed integer linear program. In Sect. 5 simulation results are shown and, finally, in Sect. 6 some conclusions are drawn.

\section{Preliminaries in Flexibility}

Before we describe the flexibility metrics, firstly, we present some preliminaries in power system flexibility as defined in [10]. Also, in Table 1 the nomenclature is displayed.

\subsection{Net Load Ramps}

The net load ramp time series $N L R_{t, i}$ for time interval $i$, at observation $t$, is defined as follows

$$
N L R_{t, i}=N L_{t}-N L_{t-i}
$$

The net load ramps can be positive or negative. Also, the two following variables are introduced

$$
\begin{aligned}
& N L R_{t, i,+}=N L R_{t, i} \forall N L R_{t, i}>0 \\
& N L R_{t, i,-}=-N L R_{t, i} \forall N L R_{t, i}<0
\end{aligned}
$$

which denote positive and negative ramping occurrence. 
Table 1. Nomenclature.

\begin{tabular}{l|l}
\hline Symbol & Definition \\
\hline$G$ & The set of the 26 generators \\
\hline$S$ & Scenario \\
\hline$T$ & The length of generation planning horizon $(24 \mathrm{~h})$ \\
\hline$t$ & Indices of thermal generators \\
\hline$S U_{g t}$ & Time period \\
\hline$S D_{g t}$ & Start up cost of unit $g$ at time $t$ \\
\hline$L_{g}$ & Shut down cost of unit $g$ at time $t$ \\
\hline$l_{g}$ & Minimum ON time of generator $g$ \\
\hline$S U t i m e_{g}$ & Minimum OFF time of generator $g$ \\
\hline$S D t i m e_{g}$ & Start up time of generator $g$ \\
\hline$N L_{t}$ & The net load at observation $t$ \\
\hline$P_{g}^{\text {max }}$ & Maximum power generation capacity for generator $g$ \\
\hline$P_{g}^{\text {min }}$ & Minimum power generation capacity for generator $g$ \\
\hline$R U_{g}$ & Ramping up limit of generator $g$ \\
\hline$R D_{g}$ & Ramping down limit of generator $g$ \\
\hline$R S_{t}$ & Spinning reserve requirement at time $t$ \\
\hline$S_{g}^{\text {max }}$ & Maximum spinning reserve of generator $g$ \\
\hline$D_{t}$ & Forecasted hourly demand at time $t$ \\
\hline$V O L L$ & Value loss of load (Euro/MWh) \\
\hline$a_{g}, b_{g}$ & Linear coefficients of fuel cost function \\
\hline$a_{g}^{\prime}, b_{g}^{\prime}$ & Linear coefficients of reserve cost function \\
\hline$u_{g t}$ & Commitment decision for unit $g$ at time $t$ \\
\hline$v_{g t}$ & Binary variable, start up action of unit $g$ at time $t$ \\
\hline$w_{g t}$ & Binary variable, shut down action of unit $g$ at time $t$ \\
\hline$p_{g t}$ & The thermal power generation output of unit $g$ at time $t$ \\
\hline$s_{g t}$ & Spinning reserve of unit $g$ at time $t$ \\
\hline$\Delta_{t}$ & Load-shedding loss at time $t$ \\
\hline & \\
\hline$S O$
\end{tabular}

\subsection{Available Resource Flexibility}

Given the operating state of a unit $g$ we can calculate the available upward and downward flexibility it can offer. In particular, the available upward (+) flexibility $\left(\right.$ Flex $\left._{t, g, i,+}\right)$, for a resource $g$, over a horizon $i$, at observation $t$, is defined as

$$
\text { Flex }_{t, g, i,+}=R U_{g} *\left(i-\left(1-u_{g t}\right) * \text { SUtime }_{g}\right) .
$$


Upward flexibility is bounded by the maximum production capacity of the resource. Also, when the resource is offline, its start up time must be less than the considered time horizon, and also it must have sufficient time to reach minimum stable production. These two aforementioned conditions can be expressed mathematically as follows

$$
\begin{gathered}
p_{g t}+\text { Flex }_{t, g, i,+} \leq P_{g}^{\max } . \\
p_{g t}+\text { Flex }_{t, g, i,+} \in \mathbb{R}-\left(0, P_{g}^{\mathrm{min}}\right) .
\end{gathered}
$$

The downward (-) flexibility available $\left(\right.$ Flex $\left._{t, g, i_{-}-}\right)$from a unit $g$ can be in a similar manner calculated as

$$
\text { Flex }_{t, g, i,-}=R D_{g} * u_{g t} .
$$

The downward flexibility available is constrained by the minimum stable production level, i.e. the unit must either operate at least at minimum production level or shut down completely. The constraints for the downward flexibility available can be expressed mathematically as follows

$$
\begin{gathered}
p_{g t}-\text { Flex }_{t, g, i,-} \geq 0 \\
p_{g t}-\text { Flex }_{t, g, i,-} \in \mathbb{R}-\left(0, P_{g}^{\mathrm{min}}\right) .
\end{gathered}
$$

\subsection{System Flexibility}

Addition of the available flexibility of each individual unit results in the total system flexibility

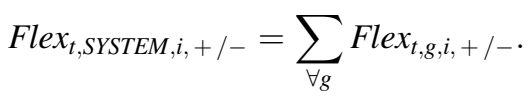

\section{Flexibility Metrics}

Now, we are ready to introduce the flexibility metrics utilized in this work.

\subsection{IRRE}

The available flexibility distribution $\left(A F D_{i,+-}(X)\right)$ can be calculated with the empirical cumulative distribution function of the flexibility available $X$. The $A F D_{i,+-}(X)$ represents the probability that $X \mathrm{MW}$, or less, of flexible resource will be available during the $i$ time horizon. The probability that the system has insufficient ramp resources at each 
observation, over each time horizon and direction, are calculated from the cumulative probability. In order to exclude these cases when the flexibility available is equal to the net load changes, the net load ramps are reduced to a value just below the net load value (for example $1 \mathrm{MW}$ ). Therefore, the insufficient ramping resource probability (IRRP) at observation $t$, over a horizon $i$, is:

$$
\operatorname{IRRP} P_{t, i,+/-}=A F D_{i,+/-}\left(N L R_{t, i,+/-}-1\right) .
$$

The sum of the IRRP values over the entire time series, $T_{+/-}$, for each direction results in the IRRE, as defined in the following equation

$$
I R R E_{i,+/-}=\sum_{\forall t \in T_{+/-}} \operatorname{IRRP}_{t, i,+/-} .
$$

\subsection{The Proposed Metric}

Given the total available flexibility of the power system and the flexibility requirements calculated from the net load ramps, we can calculate the flexibility residual of the system at each observation $t$ and time horizon $i$, for both directions (upward and downward) as follows

$$
\begin{gathered}
\text { FlexResidual }_{t, S Y S T E M, i,+}=\text { Flex }_{t, S Y S T E M, i,+}-\max \left\{N L R_{t, i,+}, 0\right\} \\
\text { FlexResidual }_{t, S Y S T E M, i,-}=\text { Flex }_{t, S Y S T E M, i,-}-\max \left\{N L R_{t, i,-}, 0\right\} .
\end{gathered}
$$

In order to calculate the probability distribution function of the flexibility residual time series we fit a non-parametric kernel distribution [12]. This way we avoid making any assumption about the underlying distribution of the time series. Given a random sample $x_{1}, x_{2}, \ldots, x_{n}$ from an unknown distribution, the kernel density estimator is given by

$$
\widehat{f_{h}(x)}=\frac{1}{n h} \sum_{i=1}^{n} K\left(\frac{x-x_{i}}{h}\right)
$$

where $\widehat{f_{h}(x)}$ the estimated probability density function, $K$ the kernel function, $n$ the sample size and $h$ the bandwidth. The kernel function and the bandwidth are the parameters that need to be selected. For this study we selected the Gaussian Kernel, which is the most often used and is given as follows

$$
K(x)=\frac{1}{\sqrt{2 \pi}} \exp \left(-\frac{1}{2} x^{2}\right) .
$$


The parameter $h$ needs tuning in order to avoid over fitting or under fitting the underlying time series, which was selected according to Silverman's rule of thumb [13]. Finally, by calculating the probability of the flexibility residual being less than zero, we determine the probability that the system has insufficient ramp resources.

\section{Unit Commitment Problem Formulation}

In this section we provide a brief description of the unit commitment problem and the constraints included in this study. The problem is formulated as a mixed integer linear program (MILP), programmed using the YALMIP toolbox [14] and solved with a commercial solver. To minimize the total generation cost of the system the following deterministic unit commitment problem for energy and ancillary services co-optimization is formulated as in [15] where the objective is the following

$$
\begin{aligned}
& \min \sum_{g \in G} \sum_{t \in T}\left(S U_{g t} v_{g t}+S D_{g t} w_{g t}\right)+\sum_{t \in T} \sum_{g \in T}\left[\left(b_{g} p_{g t}+a_{g} u_{g t}\right)+\left(b_{g}^{\prime} s_{g t}+a_{g}^{\prime} u_{g t}\right)\right] \\
& \quad+\operatorname{VOLL} \sum_{t \in T} \Delta_{t}
\end{aligned}
$$

which minimizes start-up costs, shut-down costs and generation costs, using a linear approximation of the fuel cost function. Equations (18) and (19) represent the constraints that affect the unit commitment decision. In particular, the minimum ON and OFF time constraints are given by

$$
\begin{aligned}
& u_{g t}-u_{g(t-1)} \leq u_{g \tau} \forall g \in G, t \in T, \tau=t, \ldots, \min \left\{t+L_{g}-1,|T|\right\} \\
& u_{g(t-1)}-u_{g t} \leq 1-u_{g \tau} \forall g \in G, t \in T, \tau=t, \ldots, \min \left\{t+l_{g}-1,|T|\right\}
\end{aligned}
$$

where $\tau$ is a possible operating time period starting from time $t$, while the start up and shut down action constraints can be expressed as

$$
\begin{aligned}
& v_{g t} \geq u_{g t}-u_{g(t-1)} \forall g \in G, t \in T \\
& w_{g t} \geq-u_{g t}+u_{g(t-1)} \forall g \in G, t \in T .
\end{aligned}
$$

The level of generation and spinning reserve provided by each unit is determined by the following constraints $\forall g \in G, t \in T$

$$
\begin{gathered}
\sum_{g \in G} p_{g t}+\Delta_{t} \geq D_{t} \\
P_{g}^{\min } u_{g t} \leq p_{g t} \leq P_{g}^{\max } u_{g t} \\
p_{g t} \geq 0
\end{gathered}
$$




$$
\begin{gathered}
p_{g t}-p_{g(t-1)} \leq P_{g}^{\min }\left(2-u_{g t}-u_{g(t-1)}\right)+R U_{g}\left(1+u_{g(t-1)}-u_{g t}\right) \\
p_{g(t-1)}-p_{g t} \leq P_{g}^{\min }\left(2-u_{g t}-u_{g(t-1)}\right)+R D_{g}\left(1-u_{g(t-1)}+u_{g t}\right) \\
\sum_{g \in G} s_{g t} \geq R S_{t} \\
0 \leq s_{g t} \leq S_{g}^{\max } u_{g t} .
\end{gathered}
$$

Equations (20) and (25) are constraints concerning the total system generation and reserve based on the net load forecast, while the remaining constraints are based on techno-economic parameters of each plant, including generation output, ramp rate and reserve provision. For this study we assumed that the net load is perfectly forecasted in each scenario and that perfect market completion exists, thus the generation bids represents the marginal cost of each unit. Also, only spinning reserves were considered.

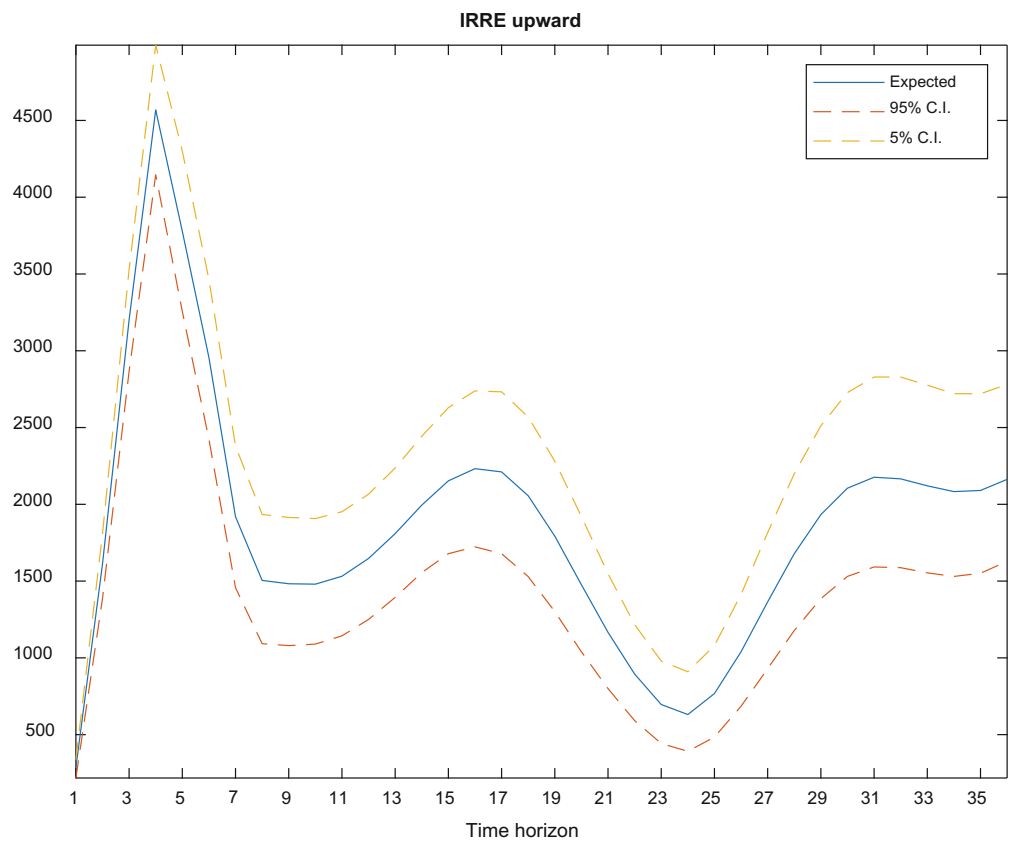

Fig. 1. The expected value of the IRRE metric and its confidence interval for the time period 2020-2024 for the Greek power system. 


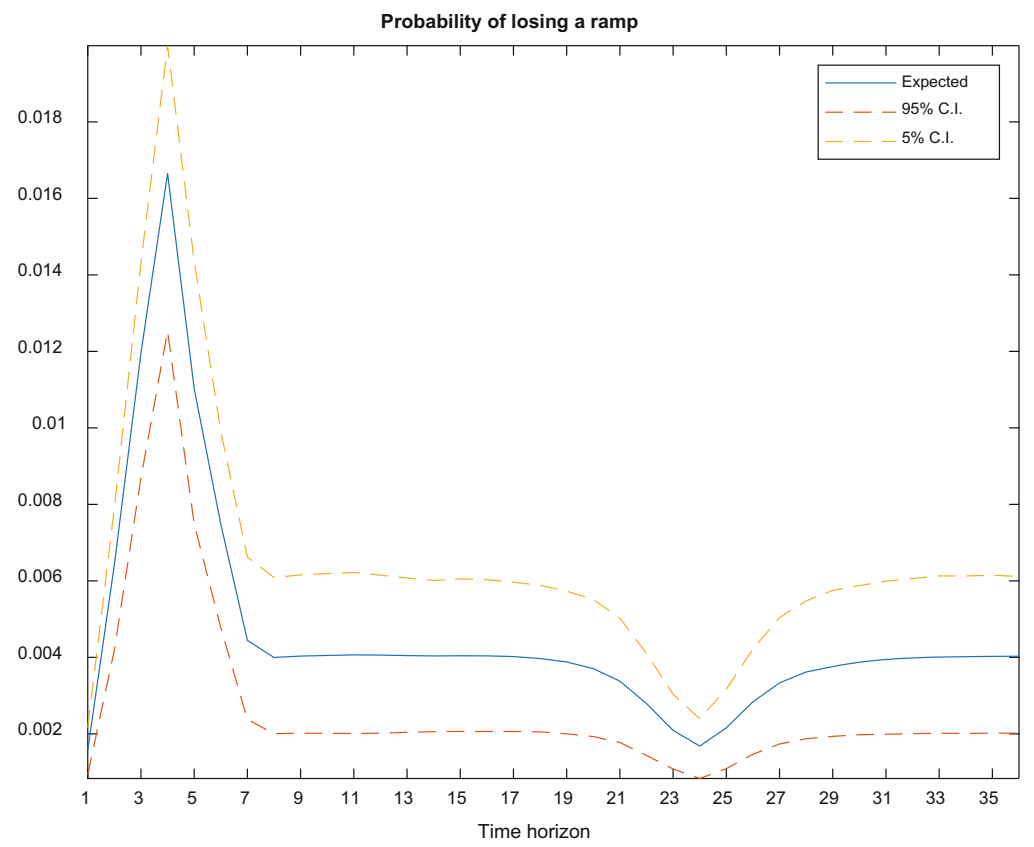

Fig. 2. The expected value of the proposed metric and its confidence interval for the time period 2020-2024 for the Greek power system.

\section{Results}

For the case study we used data from the Greek power system which are utilized in the Mid-term Adequacy Forecast conducted by ENTSO-E [16]. The different demand forecast scenarios examined in the case study correspond to various climate years for the time period 2020-2024, while the base case scenario both in demand growth and VRE penetration and normal scenario for hydro production are assumed. These scenarios are determined based on the methodology provided in [16].

The operation of thermal generators is modeled in detail, using all the technoeconomic data available (ramp rates, generation constraints, marginal costs, etc.), while the maintenance schedule of thermal units considered for this study is the same as the schedule assumed in the adequacy studies of the Greek power system. Furthermore, commission of new power plants and expected retirement of old plants are also taken into account based on the Greek ten year network development plan. The operation of the hydro units is implemented for peak shaving operation for each scenario under consideration. The contribution of interconnections is regarded as the equivalent of a thermal unit of $500 \mathrm{MW}$ base load with $95 \%$ availability. Although this is a conservative approach, since Greece is highly dependent from interconnections, this will enable us to remain on the safe side. Finally, the demand side management, since it is considered a safety measure and does not participate in the market, it is used only in cases of emergency as a last resort in the simulation study. 
After taking into account all the examined scenarios, the expected values of the IRRE index and the proposed metric for different time intervals are determined along with two extreme values of the confidence interval. The results for both metrics for the case of upward ramps for the time period 2020-2024 are shown in Figs. 1 and 2. It is evident that the calculated metrics produce similar results, as they both identify the 5-h time horizon as the most vulnerable to lose a ramping event. By comparing the rest of the time horizons, the IRRE displays also a larger value in the 16-h time horizon in contrast to our proposed metric. This can be attributed to the fact that IRRE does not take into consideration the temporal correlation between the ramping events and the available flexibility at the particular observation, but rather compares them ex-post, which in turn may lead to falsely identifying particular time horizons as more in risk than they actually are. For example, in the time frame from 5:00 am to 9:00 pm the power system will experience the full extent of variability in the net load, resulting in very large upward ramps, but also in that time frame the system is able to provide a lot of upward flexibility since most units at 5:00 am are operating at the technical minimum (base units) or are offline (peak units). This underlines the benefit of modeling the probability of losing a ramp based on the flexibility residual.

Furthermore, as one can observe from Fig. 1, the expected value of the IRRE metric (i.e. 4500) is used only to highlight the time horizons that are at most risk rather than to determine the absolute level of risk. On the contrary, the metric proposed in this paper provides realistic values of the probability of the system having insufficient ramp resources in the different time horizons. In particular, our methodology shows that there is a probability ranging from under 0.012 to over 0.020 of losing an upward ramp in the 5 -h horizon for the various scenarios.

\section{Conclusions}

In this paper, an index based on Kernel density estimators is proposed that can measure accurately the flexibility of a power system. Calculating the metric and comparing it with the IRRE index in the unit commitment problem for the Greek power system, we showcased that the proposed metric achieves to evaluate effectively the flexibility of the system in the long term horizon. Therefore it can be a very useful tool for system operators in the planning phase and can accommodate flexibility studies.

Acknowledgments. The paper includes partial results on flexibility assessment for transmission systems in line with the FLEXITRANSTORE project. The authors are grateful to all the other partners cooperating in the project and the support received from them. This project has received funding from the European Union's Horizon 2020 research and innovation programme under grant agreement No. 774407, for which the authors wish to express their gratitude. For further information, please check the website: www.flexitranstore.eu. 


\section{References}

1. Ma, J., Silva, V., Belhomme, R., Kirschen, D.S., Ochoa, L.F.: Evaluating and planning flexibility in sustainable power systems. IEEE Trans. Sustain. Energy 4(1), 200-209 (2013)

2. International Energy Agency: Harnessing Variable Renewables, pp. 41-67. International Energy Agency, Paris (2011)

3. Calabrese, G.: Generating reserve capacity determined by the probability method. Trans. Amer. Inst. Elect. Eng. 66(1), 1439-1448 (1947)

4. Billinton, R., Allan, R.: Reliability Evaluation of Power Systems, 2nd edn. Plenum, New York (1996)

5. Billinton, R., Fotuhi-Firuzabad, M.: A basic framework for generating system operating health analysis. IEEE Trans. Power Syst. 9(3), 1610-1617 (1994)

6. Stratigakos, A.C., Krommydas, K.F., Papageorgiou, P.C., Dikaiakos, C., Papaioannou, G.P.: A suitable flexibility assessment approach for the pre-screening phase of power system planning applied on the greek power system. In: Proceedings of IEEE EUROCON 2019 18th International Conference on Smart Technologies, Novi Sad, Serbia, pp. 1-6 (2019)

7. Bresesti, P., Capasso, A., Falvo, M.C., Lauria, S.: Power system planning under uncertainty conditions. Criteria for transmission network flexibility evaluation. In: Proceedings of 2003 IEEE Bologna Power Tech Conference, Bologna, Italy, July 2003

8. Capasso,A., Falvo, M.C., Lamedica, R., Lauria, S., Scalcino, S.: A new methodology for power systems flexibility evaluation. In: Proceedings of 2005 IEEE Russia Power Tech, St. Petersburg, pp. 1-6, June 2005

9. Zhao, J., Zheng, T., Litvinov, E.: A unified framework for defining and measuring flexibility in power system. IEEE Trans. on Power Syst. 31(1), 339-347 (2016)

10. Lannoye, E., Flynn, D., O’Malley, M.: Evaluation of power system flexibility. IEEE Trans. Power Syst. 27(2), 922-931 (2012)

11. Lannoye, E., Flynn, D., O’Malley, M.: Assessment of power system flexibility: a high-level approach. In: Proceedings of 2012 IEEE Power and Energy Society General Meeting, San Diego, CA, pp. 1-8 (2012)

12. Bowman, A.W., Azzalini, A.: Applied Smoothing Techniques for Data Analysis. Oxford University Press Inc., New York (1997)

13. Silverman, B.W.: Density Estimation for Statistics and Data Analysis. Chapman \& Hall/CRC, London (1986)

14. Lofberg, J.: YALMIP: a toolbox for modeling and optimization in MATLAB. In: Proceedings of IEEE International Symposium on Computer Aided Control Systems Design (2004)

15. Huang, Y., Pardalos, P.M., Zheng, Q.P.: Electrical power unit commitment: deterministic and two-stage stochastic program-ming models and algorithms. Springer (2017)

16. ENTSO-E: Mid-Term Adequacy Forecast 2018 Edition, Brussels (2018) 
Open Access This chapter is licensed under the terms of the Creative Commons Attribution 4.0 International License (http://creativecommons.org/licenses/by/4.0/), which permits use, sharing, adaptation, distribution and reproduction in any medium or format, as long as you give appropriate credit to the original author(s) and the source, provide a link to the Creative Commons license and indicate if changes were made.

The images or other third party material in this chapter are included in the chapter's Creative Commons license, unless indicated otherwise in a credit line to the material. If material is not included in the chapter's Creative Commons license and your intended use is not permitted by statutory regulation or exceeds the permitted use, you will need to obtain permission directly from the copyright holder.

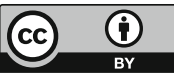

